\title{
MRP with imprecise demand and uncertain lead time
}

\author{
Romain GUILLAUME ${ }^{1,2}$ Caroline THIERRY ${ }^{1}$ Bernard GRABOT $^{2}$ \\ ${ }^{1}$ Université de Toulouse IRIT / UTM 5, Allées A. Machado, \\ 31058 Toulouse Cedex 1 - France; guillaum@irit.fr, thierry@univ-tlse2 \\ ${ }^{2}$ Université de Toulouse INPT / LGP / ENIT 47 Av. d'Azereix, \\ BP 162965016 Tarbes Cedex - France; bernard@enit.fr
}

\begin{abstract}
Taking into account the uncertainty of real data in the planning process is a real challenge for nowadays companies. It is suggested in this communication to take into account the demand uncertainty, but also the uncertainty on the lead times, for deciding which quantities of components should be released, and when. In that purpose, the various situations that may happen are summarized in a graph which design is detailed here.
\end{abstract}

Keywords: MRP, lead time, Possibility theory, graph

\section{Problem statement and state of art}

In an industrial context, data are often imprecise or uncertain. In production management, it may for instance be the case for the demand, the lead times, the resources required, their capacities, the transportation times, the inventory or production costs, etc. When analyzing the state of the art on this subject, it can be see that the uncertainty on the demand is a great focus of the literature, while the uncertainty on costs and capacities is also often considered. On the other hand, the uncertainty on the lead times, which is often mentioned [1] and may have an important impact on the performance of the Supply Chains, is quite seldom taken into account.

Various approaches can be used for dealing with uncertainty in this context. When uncertainty is modeled by possibility distributions, a first approach consists in using a function aiming at ranking fuzzy numbers in order to allow the decision maker to defuzzify imprecise values [2] [3] [4]: we will denote such approaches as "defuzzification approaches". After the defuzzification process, the result is a classical linear optimization system.

A second approach, based on the fact that a possibility distribution can be seen as a set of probability distributions, consists in choosing one of those probability distributions, according to the attitude of the decision maker (e.g. pessimistic using necessities, optimistic through possibilities, etc.) [5]. Once this choice has been made, it is possible to use a stochastic optimization model. So, the decision maker chooses for which probability distribution his solution will be optimal.

A third approach is possibilistic optimization [6], which tries to find the solution which minimizes the cost and maximizes the possibility level of a considered scenario. In other terms, this approach finds a "possibly optimal" solution; the level of possibility that the considered scenario will happen is therefore maximal.
A fourth approach is decision support ([7], [8]) based on decision support tools in which the uncertainties are propagated within the model until a decision has to be made. These decisions may for instance concern production lot sizes, capacity, sub-contracting etc. The advantage of this approach compared to the previous ones is that the user does not make an a priori decision on uncertain values, but can assess the consequences of the uncertainty on the system, through uncertainty propagation.

Another approach, denoted "robust optimization" [9], is close to the third one: by taking into account the various scenarios with their respective levels of possibility, it aims at minimizing either the maximum cost on the whole set of scenarios (minmax) or, on the whole set of scenarios, the difference between the cost of the optimal solution and the one of the proposed one (minmax Regret). In spite of its interest and to our knowledge, this approach has not yet been applied on the Lot Sizing Problems (LSP) when uncertainties are modeled through possibility distributions.

On the base of the gaps identified in the previous short state of the art, we suggest a method for computing gross requirements taking into account the uncertainty on the demand and on the lead times, within a decision support approach. The result of this method is a set of plans represented in a graph in which each level denotes a period, a path defining a scenario. A possibility distribution representing the possible quantities to process during a period is attached to each node. Each arc is valued by the possibility level that the link exists.

This graph may be used for calculating robust release policies or, for critical products, in a decision support method allowing the decision maker to choose iteratively the quantities to produce, on the base of several indicators and proposals, taking into account the levels of possibility/necessity to require these quantities.

\section{MRP and uncertainty}

\subsection{Crisp MRP}

The MRP2 method (MRP standing for Manufacturing Requirement Planning) is a comprehensive production management method allowing to build a supply planning and a production planning on the base of forecasts, using the Bill of Materials (BOM) of the products. Within this method, the MRP step (MRP standing here for Material Requirement Planning) is the step where gross then net requirements are generated for each component of the BOM, on the base of the Master Production Planning [10]. For simplification reasons, we shall here focus on a single 
level of the MRP calculation, i.e. on a single level of the $\mathrm{BOM}$, but the process is the same when several levels are considered.

Algorithm 1 shows how the Net Requirements can be calculated for a given level of the BOM.

Algorithm 1: Net requirement calculation for a given
level of the BOM

Index

$\mathrm{s}$ : index of the scenario

$\mathrm{t}$ : period

Input

$G R_{t}$ : the Gross Requirement for each period,

$I_{t 0}:$ the inventory level of the period $t 0$

$S R_{t}$ : the scheduled receipt of the period $t$

$\mathrm{d}$ : lead time

\section{Dependant variables}

$I_{t}:$ the inventory level of the period $t$

\section{Output:}

$N R_{t}$ : Net Requirements of the period $\mathrm{t}$

$N R_{t}$ : Planned order release of the period $\mathrm{t}$

$$
\begin{aligned}
& \text { For } t=1 \text { to } T \text { do } \\
& N R_{t}=G R_{t}-S R_{t}-I_{t} \\
& R_{t}=N R_{t+d}
\end{aligned}
$$

\section{End For}

\subsection{Representation of imperfect MRP data}

Among the numerous data required by the MRP calculation, several may be pervaded by uncertainties. In the framework of this study, we shall consider more specifically uncertain lead times and an imprecise demand. The information about the uncertainty of the lead time and about the imprecision of the demand is obtained from an expert knowledge. To formalize this information, the possibility theory is used in this communication. theory.

Let us first recall some results from the possibility

The uncertainty of all events A from a subset $\mathrm{S}$ can be represented by two levels:

- the possibility that the event occur (high level): possibility $\Pi(A)$,

- the necessity that the event occur (low level): necessity $\mathrm{N}(A)$,

with

$$
\forall A \subseteq S, \mathrm{~N}(A) \leq \prod(A)
$$

and

$$
\forall A \subseteq S, \mathrm{~N}(A)=1-\Pi(\bar{A})
$$

If $A$ is certainly true $\Pi(A)=1$ and $\mathrm{N}(A)=1$
If $A$ is certainly false: $\Pi(A)=0$ and $\mathrm{N}(A)=0$

If there is no knowledge available: $\Pi(A)=1$ and $\mathrm{N}(A)=0$ (the event is fully possible but not necessary at all).

An imprecise information is $v \in A$ where $A$ is a subset of $S$ which contains more than one element. The imprecision may be expressed by a disjunction of values [11]. The imprecise information $v v \in A$ defines a possibility distribution on $\mathrm{S} . v \in A$ means that all values from $v$ out of $A$ are supposed to be impossible [11].

A possibility distribution $\pi_{v}$ of $v$ is a function of $S$ in $L$ such as $\forall s \in S, \pi_{v}(s) \in L$, and $\exists s, \pi_{v}(s)=1$ with $v$ denoting an ill-know value in $S, L$ being the scale of plausibility (normally, [0,1]).

When a possibility distribution is represented by a trapezoidal fuzzy set, an imprecise information is denoted by $(\mathrm{a}, \mathrm{b}, \mathrm{c}, \mathrm{d}, \mathrm{h})$ with $\mathrm{h}=1$, like in Figure 1.

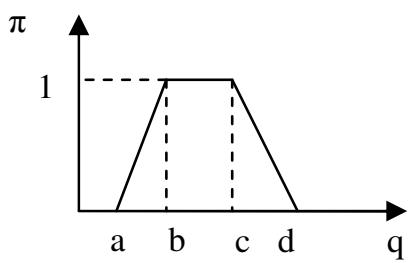

Fig. 1: Representation of a trapezoidal fuzzy set

In the case of the fuzzy MRP, the imprecision on the gross requirements can thus be represented by a possibility distribution: $\pi\left(\mathrm{Q}_{\mathrm{o}}\right)$. The uncertainty on the lead time can be represented by a possibility level for the different values of the lead time: $\Pi(\delta=a)$.

In this article, we consider that this lead time has only two values with high degrees of possibility (the others are not taken into account). This is a first improvement in comparison with the usual case where lead times are supposed to be certain. The result is that we shall have to work with possible scenarios, instead of situations supposed to be certain.

\section{Computation of the possible releases}

The objective of this communication is to suggest a method for modeling releases, in a way:

- which integrates the calculation of the possible net requirements on the base of imprecise gross requirements (fuzzy),

- which models the set of possible releases through a graph,

in order to generate robust solutions as a second step.

We consider here that the gross requirements are imprecise only on the flexible horizon, i.e. after the period on which the orders are firm. We shall as a consequence consider that a crisp calculation is performed up to the first period of this flexible horizon. The scheduled receipts are crisp.

We suggest to compute the requirement planning in three steps: 
- calculation of scenarios for fuzzy net requirements,

- design of the graph of possible releases,

- use of the graph.

\subsection{Computation of the net requirements}

In the case of fuzzy gross requirements, several scenarios of possible net requirements exist, according to the way the inventory or the scheduled receipts will be consumed. The first problem is to calculate these scenarios.

Algorithm 2 calculates the possible net requirements scenarios corresponding to the different possible ways to use the initial inventory or the scheduled receipts. Therefore, there exist as many possible scenarios as the number of periods on which this consumption by the gross requirements runs.

For each scenario (corresponding to a given number of periods of consumption):

- the net requirements up to the last consumption period are null,

- the net requirements are calculated on the last period of consumption,

- the net requirements for the following periods are equal to the gross requirements (there is no more possible consumption).

- the possibility degree of a scenario is equal to the minimum of the possibility degrees of the inventories and net requirements on all the periods.

To compute the scenario of the fuzzy net requirements, we apply algorithm 2 .

Algorithm 2 is used iteratively to compute:

1) the different scenarios of fuzzy net requirements without considering the scheduled receipt.

2) the different scenarios of fuzzy net requirements for each of the previous scenarios and for each scheduled receipt to be consumed.

Algorithm 2: Computation of the fuzzy net requirements

Index

s: index of the scenario

t: period

\section{Input}

$G R_{t}$ : fuzzy gross requirements for each period,

$t f$ : period of the scheduled receipt to be consumed,

$I_{t f-1}$ : inventory level of period $t f-1$

$S R_{t f}$ : scheduled receipt of period $t f$

\section{Dependant variables}

$I_{t}:$ inventory possibly not consumed

\section{Output:}

$N R_{t, s}$ : fuzzy possible Net Requirements of the period $t$ for scenario $\mathrm{s}$
$\Pi_{s}$ : possibility level of scenario s.

$s=1$

$\Pi_{1}=1$

$\$ For all the last possible periods of consumption of the possible inventory

For $t=t f$ to $T$ do

$\backslash$ If there is a scheduled receipt to be consumed If $t=t f$ Then

\IScheduled receipt is integrated to the stock level $I_{t-1}=I_{t-1}+S R_{t}$

End If

$\$ As long as there can be inventory (including scheduled receipts) to be consumed

While $\Pi\left\{I_{t-1} \geq 0\right\}>0$ do

$\$ The inventory is calculated for the next period

$$
I_{t}=-\min _{q}\left(G R_{t}(-) I_{t-1} ; 0\right)
$$

$\$ If it is possible that the inventory is not sufficient

$$
\begin{gathered}
\text { If } \Pi\left\{\max _{q}\left(G R_{t}(-) I_{t-1} ; 0\right) \geq 0\right\}>0 \text { then } \\
\backslash \backslash \text { The requirement is calculated } \\
N R_{t, s}=\max _{q}\left(G R_{t}(-) I_{t-1} ; 0\right)
\end{gathered}
$$

$\$ The requirements on the following periods are equals to the gross requirements

$$
\begin{aligned}
& \text { For } i=t+1 \text { to } T \text { do } \\
& \qquad N R_{i, s}=G R_{i}
\end{aligned}
$$

\section{End For}

$\$ The possibility of the scenario is updated according to the possibility of the net requirement on this period

$$
\Pi_{s}=\min \left(\Pi\left\{N R_{t, s} \geq 0\right\}, \Pi_{s}\right)
$$

$\$ If it is possible that an inventory exists

$$
\text { If } \Pi\left\{I_{t} \geq 0\right\}>0 \text { then }
$$

I\A new scenario is created

$$
\mathrm{s}=\mathrm{s}+1
$$

$\$ The possibility of the scenario is updated according to the possibility of the inventory on this period

$$
\Pi_{s}=\min \left(\Pi\left\{I_{t} \geq 0\right\}, \Pi_{s}\right)
$$

IVFor this new scenario, all the requirements up to the last period of consumption of the last considered scenario are null

$$
\begin{aligned}
& \text { For } i=0 \text { to } t \text { do } \\
& \qquad N R_{i, s}=0
\end{aligned}
$$

\section{End For}

\section{End If}

\section{End If}

$$
I=I_{t}
$$

\section{End While}

\section{End For}

// If the inventory is sufficient for the whole horizon whatever the gross requirement is.

$$
\begin{aligned}
& \text { If } N\left\{I_{T} \geq 0\right\}>0 \text { Then } \\
& \text { For } i=0 \text { to } t \text { do } \\
& \qquad N R_{i, s}=0
\end{aligned}
$$




\section{End If}

\section{Example:}

Remark: The notation for the fuzzy number $(a(\alpha) ; b ; c ; d)$ represents the fuzzy number illustrated by Figure 2 .

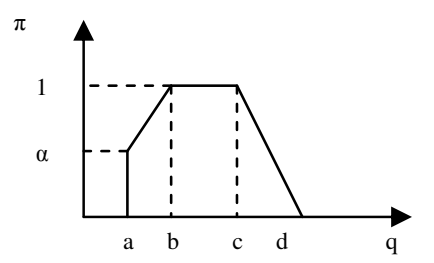

Fig. 2: Illustration of a fuzzy number $(a(\alpha) ; b ; c ; d)$

Let us consider $\mathrm{tf}=3, \mathrm{~T}=6, \mathrm{I}_{\mathrm{tf}-1}=15$,

GR3 $=(5 ; 10 ; 15 ; 20) \quad$ GR4 $=(5 ; 10 ; 15 ; 20)$

GR5 $=(5 ; 10 ; 15 ; 20) \quad$ GR6 $=(5 ; 10 ; 15 ; 20)$.

There is no scheduled receipt after period 3 .

Let us apply the algorithm 2 to these data:

We compute $\max _{q}\left(G R_{3}(-) I_{2} ; 0\right)=(0,0,0,5)$ and

$I_{3}=(0 ; 0 ; 5 ; 10)$.

Since $\max _{q}\left(G R_{3}(-) I_{2} ; 0\right)>0$,

$N R_{3,1}=(0 ; 0 ; 0 ; 5), N R_{4,1}=(5 ; 10 ; 15 ; 20), N R_{5,1}=(5 ; 10 ; 15$;

$20), N R_{6,1}=(5 ; 10 ; 15 ; 20)$ and $\Pi_{1}=1$

$I_{3}>0$ so, we create a new scenario with $N R_{3,2}=(0 ; 0 ; 0 ; 0)$

We compute the $\max \left(G R_{4}(-) I_{3} ; 0\right)=(0(0.5), 5,15,20)$ and

$I_{4}=(0 ; 0 ; 0(0.5) ; 5)$

Since $\max _{q}\left(G R_{4}(-) I_{3} ; 0\right)>0$,

$N R_{3,2}=(0 ; 0 ; 0 ; 0), N R_{4,2}=(0(0.5) ; 5 ; 15 ; 20), N R_{5,2}=(5 ; 10$

$; 15 ; 20), N R_{6,2}=(5 ; 10 ; 15 ; 20)$ and $\Pi_{2}=1$
$I_{4}>0$ so we create a new scenarios with $N R_{3,3}=(0 ; 0 ; 0 ; 0)$ $N R_{4,3}=(0 ; 0 ; 0 ; 0)$

We compute $\max _{q}\left(G R_{5}(-) I_{4} ; 0\right)=(0,10,15,20)$ and $I_{5}=(0 ; 0 ; 0 ; 0)$.

Since $\max _{q}\left(G R_{5}(-) I_{4} ; 0\right)>0$,

$N R_{3,3}=(0 ; 0 ; 0 ; 0), N R_{4,3}=(0 ; 0 ; 0 ; 0), N R_{5,3}=(0 ; 10 ; 15$;

$20), N R_{6,3}=(5 ; 10 ; 15 ; 20)$ and $\Pi_{3}=0.5$

We have three scenarios for the net requirements, represented on Figure 3.

\subsection{Computation of planned order release}

We shall consider here that the release policy is "lot for lot" (i.e. the released quantity is the quantity required for the period), since this policy is quite usual in nowadays companies. In any case, if another policy is used, its use would only result in grouping the lots expected for several periods.

Since fuzzy lead times are used, a large number of possible scenarios exist for releasing orders with a lot for lot policy: for each net requirement on a given period, two scenarios of planned order release are possible, according to the lead time which has here two possible values (it can so be seen that increasing the number of possible planned order could lead to some combinatorial difficulties).

As explained above, we have chosen here to represent the set of these possible scenarios by a graph, each level of the graph representing a period, and each path a possible scenario.

Scenario 1

Scenario 2

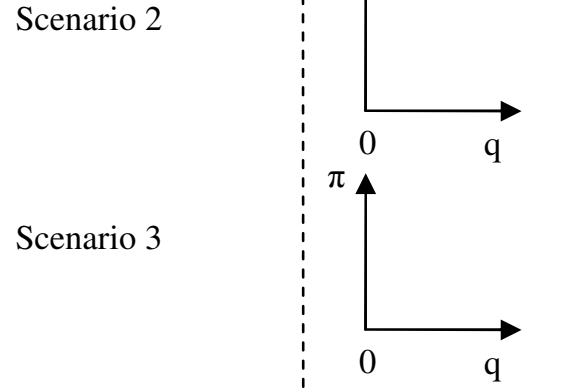

Period 3

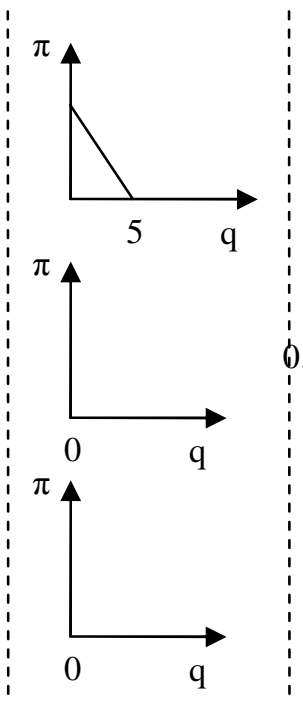

\section{Period 3}

Fig. 3: Representation of the three possible Net Requirement scenarios

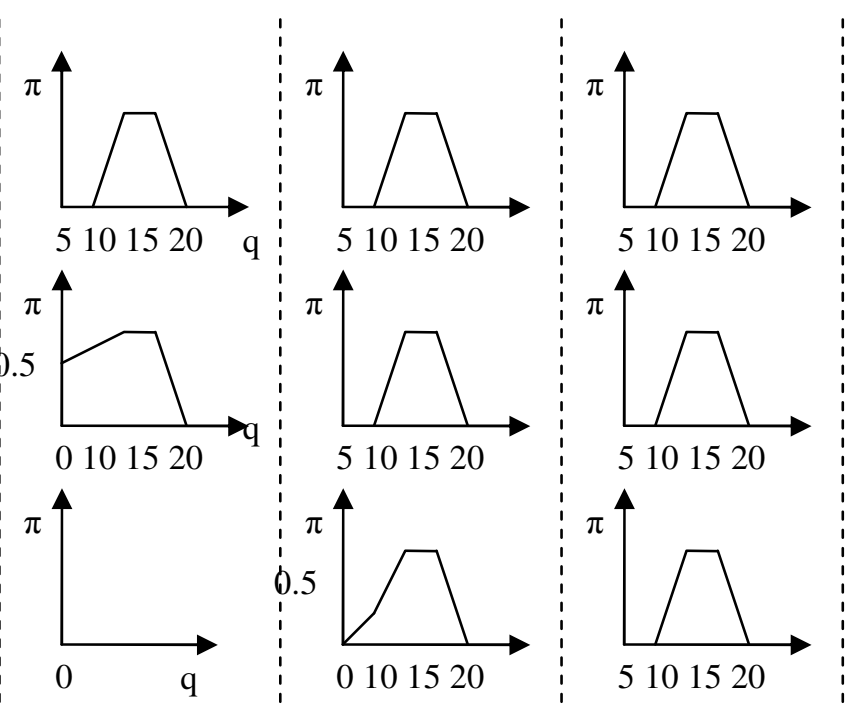

Period 6 


\subsection{Computation of the nodes}

The planned order release nodes include three types of information:

- characterization of the node (net requirements which are released within the period, expressed as a vector which gives, for each possible value, each of the possible lead times if the net requirement corresponding to this lead time is released),

- fuzzy quantity of the planned order release,

- possibility level of the node.

The characterisation of a node $(\mathrm{j}, \mathrm{t}, \mathrm{s})$ is expressed as a vector $c_{j \bullet}$ of the matrix $c_{j i}$ of the possible combination $j$ of the possible lead time $\left\{a_{i}\right\}$ with $a_{i} \in\left\{\delta_{i}, 0\right\}$. For example, if we have two possible values of $\delta_{i}$, the matrix is $\left(\begin{array}{l}0,0 \\ 0, \delta_{2} \\ \delta_{1}, 0 \\ \delta_{1}, \delta_{2}\end{array}\right)$.

A planned order release requirement node $R_{t, j, s}$ is also characterized by a vector $c_{j}$. which, a fuzzy quantity $\left(a_{j, t, s} ; b_{j, t, s} ; c_{j, t, s} ; d_{j, t, s}\right)$ and a possibility level to have the combination $j: \Pi_{j}$

For each possible lead time $\delta_{i}$ we know the possibility level of occurrence $\Pi\left(\delta_{i}\right)$ and the possibility level of nonoccurrence $\Pi\left(\overline{\delta_{i}}\right)=\max _{j}\left(\Pi\left(\delta_{j}\right) \mid j \neq i\right)$.

From these possibility levels and from the combination matrix of the possible lead times, we compute (algorithm 3 ) the possibility level of each combination $j$ as follows:

Algorithm 3: Computation of the possibility level of each combination of the possible lead time

\section{Input:}

$c_{j, i}:$ the matrix of the possible combination $j$

of the possible lead time $\left\{a_{i}\right\}$ with

$a_{i} \in\left\{\delta_{i}, 0\right\}$.

$\Pi\left(\delta_{i}\right)$ the possibility level of the realisation of

Output:

the lead time $\delta_{i}$

$\Pi_{j}:$ the possibility level of the combination $j$

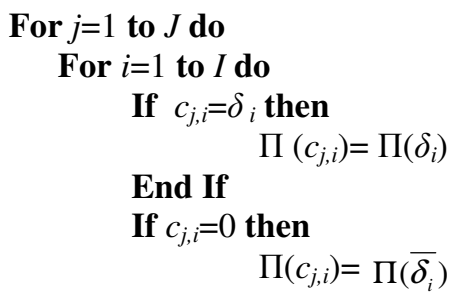

\section{End For}

$$
\Pi_{j}=\min _{i}\left(\Pi\left(c_{j, i}\right)\right)
$$

End For
We shall now, using algorithm 4, build the graph of the possible fuzzy released quantities. In order to calculate these fuzzy quantities, we add, for each period and each scenario, the net requirements corresponding to the matrix of the possible combinations.

Algorithm 4: Computation of the possible fuzzy release quantities

Input: $N R_{t, s}$ : the set of fuzzy possible Net Requirements.

$c_{j, i}$ : the matrix of possible combination $j$ of the possible lead time $\left\{a_{i}\right\}$ with $a_{i} \in\left\{\delta_{i}, 0\right\}$.

Output: $R_{t, j, s}$ : the release fuzzy quantity of the nodes $(j, t)$ of the scenario $s$.

For $s=1$ to $S$ do

For $t=t f$ to $T$ do

$\backslash$ for each combination of the possible lead times

For $j=1$ to $J$ do

$$
R_{t, j, s}=(0 ; 0 ; 0 ; 0)
$$

For $i=1$ to $I$ do

Ilwe compute the release quantity

$$
\text { If } c_{j, i}>0 \text { then }
$$

$\backslash$ as long as the net requirements of the combination belong to the horizon, we calculate the released quantity. Otherwise, we give the value $(-1 ;-1 ;-1 ;-1)$ to the quantity.

While $R_{t, j, s} \neq(-1 ;-1 ;-1 ;-1)$ do

$$
\begin{aligned}
& \text { If } t+\delta_{i} \in[t f ; T] \text { then } \\
& R_{t, j, s}=N R_{t+\delta_{i}, s}(+) R_{t, j, s} \\
& \text { Else } \\
& R_{t, j, s}=(-1 ;-1 ;-1 ;-1)
\end{aligned}
$$

\section{End If}

\section{End While}

\section{End If}

\section{End For}

\section{End For}

End For

\section{End For}

\subsection{Computation of the arcs}

Using algorithm 5, we shall then connect the compatible nodes by associating to each link the possibility that the link exists.

A requirement can only be released once. So, two consecutive releases are incompatible if the same requirement is concerned in the two releases. Two successive nodes will as a consequence be linked only if they are compatible. We give then to the arc the level of possibility of the following node. 
Note: the beginning and the end of the graph are subject to a specific processing. It appears that some nodes are not linked to the graph, which induces modifications on the possibilities of the arcs (an arc of possibility 1 must always start from a given node).

\section{Algorithm 5: Computation of the arcs}

Input: $R_{t, j, s}$ the release fuzzy quantities of the nodes $(j, t)$ of the scenario $c_{j, i}$ : the matrix of the possible combinations $j$ of the possible lead times $\left\{a_{i}\right\}$ with $a_{i} \in\left\{\delta_{i}, 0\right\}$. $\Pi_{j}$ : the possibility level of the combination $j$

Output: $\Pi_{R}^{S}$ the transition matrix of the release graph for scenario $s$.

For $s=1$ to $S$ do

$\Pi_{R}^{s}=0$

For $t=t f$ to $T$ do

For $j=1$ to $J$ do

$$
\begin{gathered}
\text { For } k=1 \text { to } J \text { do } \\
\operatorname{comp}=1 \\
\text { For } i=2 \text { to } I \text { do }
\end{gathered}
$$

IIfor each lead time $i$, we check if the combination $k$ is incompatible and if the corresponding released quantity of the nodes exists.

$$
\begin{aligned}
& \text { If } c_{j, i}=c_{k, i-1} \text { then } \\
& \text { comp }=0 \\
& \text { End If }
\end{aligned}
$$

End For

II we check if the combination is compatible and if the corresponding release quantity of the nodes exists.

\section{then}

$$
\text { If comp }=1 \&\left(R_{t, j, s} \& R_{t+1, k, s}\right) \neq(-1 ;-1 ;-1 ;-1)
$$

$\backslash$ give the possibility level to the arc between node $(j, t)$ node $(k, t+1)$

$$
\begin{aligned}
& \qquad \Pi_{R}^{s}(j, k, t)=\Pi_{k} \\
& \text { End If } \\
& \text { End For } \\
& \text { End For } \\
& \backslash \text { modifies the levels of possibility of the nodes having }
\end{aligned}
$$
only one output arc.

$$
\begin{aligned}
& \text { If } \exists \Pi_{R}^{s}(j, k, t) \neq 0 \& \Pi_{R}^{s}\left(j, k^{\prime}, t\right)=0 \forall k^{\prime} \neq k \text { then } \\
& \Pi_{R}^{s}(j, k, t)=1
\end{aligned}
$$

End If

End For

$\backslash$ an input node and an output node are added.

$$
\text { For } j=1 \text { to } J \text { do }
$$

$$
\begin{gathered}
\text { If }\left(R_{1, j, s}\right) \neq(-1 ;-1 ;-1 ;-1) \text { then } \\
\Pi_{R}^{s}(e, j, 1)=\Pi_{k}
\end{gathered}
$$

$$
\text { End If }
$$$$
\text { If }\left(R_{T, j, s}\right) \neq(-1 ;-1 ;-1 ;-1) \text { then }
$$

$$
\Pi_{R}^{s}(j, s, T+1)=1
$$

\section{End If}

\section{End For}

\section{End For}

\section{Example:}

Let us consider the first scenario of the net requirements with $\Pi(\delta=1)=0.2$ and $\Pi(\delta=2)=1$.

Thus, the matrix of the possible combinations is $\left(\begin{array}{l}1,0 \\ 1,2 \\ 0,0 \\ 0,2\end{array}\right)$

We can compute the related graph of the planned order release (Figure 4).

\subsection{Exploitation of the graph}

The graph is a general model of the planned order release under fuzzy uncertainty. From this graph, different types of information can be extracted:

- the planned order releases which have a given possibility level, by selecting the path which has this possibility [12], for instance the most possible ones;

- the possibility to release each quantity, which can also be computed by merging the nodes ([13]);

- the performances of various policies of lot sizing in presence of uncertainties, if a graph is created for each policy.

Since this communication mainly concern the visualization of several released situations through a graph, these methods are not developed in the present paper. Nevertheless, such graph can be a real support for decision making ([12], [13]). 


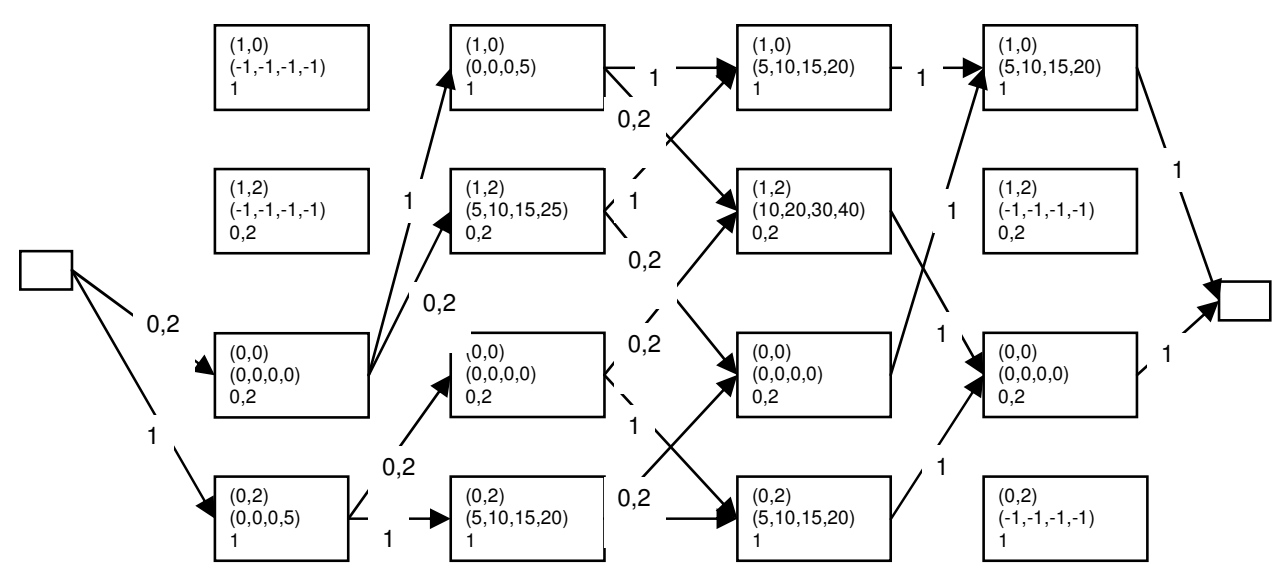

Fig. 4. Example of graph of the possible fuzzy released quantities

\section{Conclusion and perspectives}

We have suggested in this communication to take into account the uncertainty on the demand, but also on the lead times, for defining released orders. In such case, a problem is often the combinatorial explosion of the situations to consider. The suggested method also suffers from this problem: it has been seen that considering more possible lead times could lead to very complex graphs. Nevertheless, we do think that taking into accounts two or three possible lead times would already be very useful in practical cases, allowing for instance to describe several alternatives for processing the parts.

In the literature, the approach by defuzzification takes into account uncertainty on the lead time with the imprecision on the demand, but this approach deffuzzifies immediately: therefore, the decision maker knows only one scenario and does not have any information on the set of possible scenarios of released quantity.

The others approaches (possibilistic optimization, robust optimization) could be used to choose a crisp released quantity from the graph; in other words, each approach can be considered as a lot sizing rule. In "crisp" MRP, rules for lot sizing are commonly used, like economic quantity, lot for lot, etc. In a fuzzy MRP which takes into account the uncertainty on the lead time and the imprecision on the demand, the rules may be considered as optimistic lot sizing (possibilistic optimization) or pessimistic lot-sizing (robust optimization).

The suggested graph could also provide a real risk assessment of the consequences of possible hazards on the lead time, considering that the possibility that the hazard occurs could be known on the various periods of the horizon. These possible applications will be considered in our future works.

\section{References}

[1] A. Dolgui and C. Prodhon, Supply planning under uncertainties in MRP environments: A state of the art, Annual Reviews in Control, 31:269--279, 2007.

[2] D. Peidro, J. Mula, R. Poler, and J. Verdegay, Fuzzy optimization for supply chain planning under supply, demand and process uncertainties, Fuzzy Sets and Systems, 160:2640--2657, 2009.
[3] T. Liang, Fuzzy multi-objective production/ distribution planning decisions with multiproduct and multi-time period in a supply chain, Computers \& Industrial Engineering, 55:676--694, 2008.

[4] T. Liang and H. Cheng, Application of fuzzy sets to manufacturing/distribution planning decisions with multi-product and multi-time period in supply chains, Expert Systems with Applications, 36:3367-3377, 2009.

[5] S. Gao-Ji and L. Yan-Kui, Fuzzy Minimum-Risk Material Procurement Planning Problem, Proceedings of Fourth International Conference on Natural Computation (ICNC '08), 629--633, 2008.

[6] J. Mula, R. Poler, and J. Garcia-Sabater, Material Requirement Planning with fuzzy constraints and fuzzy coefficients, Fuzzy Sets and Systems, 158:783-793, 2007.

[7] C. Thierry and H. Fargier, The Use of Possibilistic Decision Theory in Manufacturing Planning and Control: Recent Results in Fuzzy Master Production Scheduling, in Scheduling under fuzziness, PhysicaVerlag, 2000.

[8] B. Grabot, L. Geneste, G. Reynoso-Castillo, and S. Vérot, Integration of uncertain and imprecise orders in the MRP method, Journal of Intelligent Manufacturing, 16:215--234, 2005.

[9] B. Roy, Robustness in operational research and decision aiding: A multi-faceted issue, European Journal of Operational Research, 200:629--638, 2010.

[10] J. Orlicky and G. Plossl, Orlicky's Material Requirement Planning, McGraw Hill Text, 2nd Edition, 1994.

[11] D. Dubois and H. Prade, Représentations formelles de l'incertain et de l'imprécis. Concepts et méthodes pour l'aide à la décision 1, 11--165, 2006.

[12] R. Guillaume, C. Thierry and B. Grabot, Modelling of ill-known requirements and integration in production planning, Production Planning \& Control, 2010. doi: 10.1080/09537281003800900.

[13] R. Guillaume, C. Thierry and B. Grabot, Integration of ill-known requirement into a plan, 39th International Conference on Computer and Industrial Engineering (CIE39), July 6-8, Troyes, France, 2009. 